\title{
PRMT1 expression is elevated in head and neck cancer and inhibition of protein arginine methylation by adenosine dialdehyde or PRMT1 knockdown downregulates proliferation and migration of oral cancer cells
}

\author{
CHUN-YI CHUANG ${ }^{1,2}$, CHIEN-PING CHANG ${ }^{3}$, YU-JEN LEE ${ }^{3}$, WEI-LONG LIN ${ }^{1}$, \\ WEN-WEI CHANG ${ }^{3,4}$, JIA-SIAN WU ${ }^{3}$, YA-WEN CHENG ${ }^{5}$, HUEI LEE ${ }^{5}$ and CHUAN LI ${ }^{3,4}$
${ }^{1}$ School of Medicine, Chung Shan Medical University, Taichung; ${ }^{2}$ Department of Otolaryngology, Chung Shan Medical University Hospital, Taichung; ${ }^{3}$ Department of Biomedical Sciences, Chung Shan Medical University, Taichung;
${ }^{4}$ Department of Medical Research, Chung Shan Medical University Hospital, Taichung; ${ }^{5}$ Graduate Institute of Cancer Biology and Drug Discovery, Taipei Medical University, Taipei 110, Taiwan, R.O.C.

Received October 14, 2016; Accepted April 5, 2017

DOI: $10.3892 /$ or.2017.5737

\begin{abstract}
Protein arginine methylation is a post-translational modification that has been implicated in signal transduction, gene transcription, DNA repair and RNA processing. Overexpression or deregulation of protein arginine methyltransferases (PRMTs) have been reported to be associated with various cancers but have not been studied in head and neck cancer (HNC). We investigated the involvement of the modification in HNC using oral cancer cell lines (SAS, OECM-1 and HSC-3) and an immortalized normal oral cells (S-G). The expression levels of the predominant PRMT1 were generally consistent with the levels of asymmetric dimethylarginine (ADMA), highest in SAS and OECM1, then S-G and low in HSC-3. Upon the treatment with an indirect methyltransferase inhibitor adenosine dialdehyde (AdOx), the ADMA levels in SAS and OECM1, but not that in S-G and HSC-3, decreased significantly. SAS and OECM with high ADMA levels grew faster than HSC-3 and S-G. The growth rate of the fast growing SAS and OECM, but not that of the other two cell lines, decreased significantly upon AdOx treatment. The migration activity of SAS and HSC-3, two cell lines with migration ability also decreased after the AdOx treatment. Immunohistochemical analyses of specimens from typical HNC patients showed strong PRMT1 expression in the tumor cells compared with neighboring normal cells. Knockdown of PRMT1 in SAS cells decreased the levels of PRMT1 and ADMA-containing proteins significantly. These cells
\end{abstract}

Correspondence to: Professor Chuan Li, Department of Biomedical Sciences, Chung Shan Medical University, Taichung, Taiwan, R.O.C. E-mail: cli@csmu.edu.tw

Key words: oral cancer cells, protein arginine methylation, PRMT1, head and neck cancer, adenosine dialdehyde, migration showed decreased growth rate, reduced migration activity but increased expression of the epithelial marker E-cadherin. The present study thus provides fundamental background for evaluation of the PRMT1 gene as the therapeutic targets of HNC.

\section{Introduction}

Besides genetic mutations in oncogenes and tumor suppressor genes, the importance of epigenetic regulation for carcinogenesis has been recognized. Epigenetic regulation includes DNA methylation and histone modifications (the so called histone code) that can modulate chromosome structure for gene expression (1). Protein arginine methylation is a post-translational modification that has been implicated in signal transduction, gene transcription, DNA repair and RNA processing. Recent studies have linked this modification to carcinogenesis and metastasis (2).

According to the attachment of methyl groups to specific guanidino nitrogen atoms of arginine, the protein arginine methyltransferases (PRMTs) are divided into four different groups: type I catalyzes the formation of asymmetric $\omega-N^{G}$, $N^{G}$ dimethylarginine (ADMA), type II symmetric $\omega-N^{G}, N^{G \prime}$ dimethylarginine (SDMA), type III $\omega-N^{G}$ monomethylarginine (MMA), and type IV enzymes catalyzing the transfer of the methyl group to the $\delta$-nitrogen of arginine residues are only reported in yeast (3). Nine different human PRMTs have been identified to catalyze protein arginine methylation $(4,5)$. PRMT1, 2, 3, 4, 6 and 8 belong to the type I while PRMT5 and PRMT9 (6) to the type II PRMT. Type III activity have been reported for PRMT7 (7).

PRMTs can affect gene expression through their coactivator/corepressor activity to modify histones and regulate transcription (5). Furthermore, PRMTs can also exert their impacts by adjusting the modified substrates for different interactions, localization, function, or signaling pathways (3). Each PRMT can modify various non-histone substrate 
proteins including many RNA binding proteins (3). Critical proteins involved in various cancers such as p53 (8), estrogen receptor (9), BRCA1 (10) and EGF receptor (11) have been reported to be methylated by PRMTs.

DNA damage response (DDR) is critical for the maintenance of genome integrity to prevent cancer development. Numerous PRMT substrates such as MRE11, Rad 9, 53BP1 and DNA polymerase $\beta$, FEN1 and BRCA1 are involved in DDR (12). Protein arginine methyltransferases thus, can influence cancer development through their regulation of DDR. Protein arginine methylation also participates in many signal transduction pathways that are critical in cancer development. For example, both PRMT1 and CARM1 have been reported to play roles in the $\mathrm{Wnt} / \beta$-catenin pathway (13-16). PRMT1 methylation of Axin, a key scaffold protein for $\beta$-catenin degradation, can stabilize Axin and thus negatively regulates WNT signaling (13-16). Overall it is apparent that protein arginine methylation is important for carcinogenesis.

Overexpression of PRMTs is often associated with various types of cancer. For example, the expression level of PRMT1 and PRMT6 in cancer cells of various tissues including bladder, lung and breast are significantly higher than that in normal (17). For melanoma, only PRMT4/CARM1 was significantly induced whereas PRMT6 was reduced (18). Understanding the involvement of certain PRMTs in a specific cancer will help to make them as therapeutic targets.

Most studies related to arginine methylation and cancer are from the studies of breast (19-23) and in prostate (24,25), lung $(17,26)$, bladder (17) and colorectal cancer $(27,28)$. Head and neck cancer is very heterogeneous with malignancies arising in the oral cavity and oropharynx, nasopharynx, hypopharynx, larynx, nasal cavity and paranasal sinuses, ear, salivary glands and thyroid. Ninety percent of the HNC malignancies are squamous cell carcinomas (HNSCC). Risk factors associated with HNSCC carcinogenesis include tobacco and alcohol use, poor diet and human papilloma virus (HPV) infection (29). There have been no reports focusing on protein arginine methylation in head and neck cancer.

Oral cancer accounts for the largest group in head and neck cancers. In the present study, we thus investigated the involvement of the modification in HNC using an immortalized oral cell (S-G) and oral cancer cell lines (SAS, OECM-1 and HSC-3). PRMT1 is the enzyme that accounts for $85 \%$ of protein arginine methylation activity (30) and has been implicated in many different cancer types. We thus first examined the levels of the predominant type I enzyme PRMT1 and the modified ADMA-containing proteins in oral cancer cells. We then treated the oral cancer cells with an indirect methyltransferase inhibitor adenosine dialdehyde ( $\mathrm{AdOx}$ ) to follow the effects of arginine methylation. AdOx can accumulate the methyltransferase product inhibitor S-adenosine homocysteine (SAH) through the inhibition of SAH hydrolase. Protein arginine methylation stands for the majority of the AdOxblocked protein methylation $(31,32)$. Furthermore, AdOx has been shown to affect the proliferation and metastasis of various cancer cells (33-37). We showed that the AdOx treatment decrease the level of ADMA-containing proteins and can reduce growth and migration in oral cancer cells that express a high level of PRMT1. Knockdown of PRMT1 protein had similar effects on cell growth and migration in the SAS oral cancer cells. We further examined PRMT1 protein in HNC patient tissue sections and observed elevated PRMT1 levels in tumors.

\section{Materials and methods}

Cell culture and cell extract preparation. Oral squamous carcinoma cancer cell lines SAS and OECM1 and immortalized human gingival keratinocyte S-G cells were used in the present study. The SAS cell line was established from a poorly differentiated squamous cell carcinoma of the tongue. The OECM-1 cell line was established from OSCC cells surgically excised from a Taiwanese patient. The S-G cell is an epithelial-like cell line established from an area of clinically normal adult human attached gingiva and thus is used as a normal control. The S-G and SAS cells were grown in Dulbecco's modified Eagle's medium (DMEM; Gibco/Life Technologies), the OECM1 cells in RPMI-1640 medium (Gibco/Life Technologies), and HSC-3 cells were in DMEM/F-12 medium (Gibco/Life Technologies) with $10 \%$ fetal bovine serum (FBS; HyClone Laboratories, Inc., Logan, UT, USA) and antibiotics (100 U/ml penicillin and $100 \mathrm{ml} / \mathrm{ml}$ streptomycin) at $37^{\circ} \mathrm{C}$ in an incubator with $5 \% \mathrm{CO}_{2}$. L-glutamine $(2 \mathrm{mM})$ was supplemented to all cell lines except HSC-3 and pyruvate $(2 \mathrm{mM})$ was supplemented to the S-G cells. After reaching confluency, the cells were harvested and washed with phosphate-buffered saline (PBS, $10 \mathrm{mM} \mathrm{Na}_{2} \mathrm{HPO}_{4} ; 1.8 \mathrm{mM} \mathrm{KH}_{2} \mathrm{PO}_{4} 140 \mathrm{mM} \mathrm{NaCl}$ and $2.7 \mathrm{mM} \mathrm{KCl}, \mathrm{pH} 7.4$ ), resuspended and lysed in buffer A [50 mM Tris-HCl, pH 7.4; 150 mM NaCl; 1 mM EDTA; $1 \%$ Triton X-100; 1x complete protease inhibitor (Roche); $10 \mathrm{mM} \mathrm{NaF}$ ] by sonication. The lysate was centrifuged and the supernatant was designated as the cell extract. The protein concentration was determined by the BCA protein assay (Thermo Fisher Scientific, Waltham, MA, USA) using bovine serum albumin (BSA) as the standard.

SDS-PAGE and western blot analyses. Protein samples were separated by SDS-polyacrylamide gel electrophoresis (PAGE) and then transferred to nitrocellulose membranes (Hybond-C Extra, Gelman Sciences; Amersham Biosciences, Little Chalfont, UK) as previously described (38). The membranes were blocked in $7 \%$ skimmed dry milk in TTBS $(10 \mathrm{mM}$ Tris-HCl, pH 7.5; $100 \mathrm{mM} \mathrm{NaCl} ; 0.1 \%$ Tween-20) for $1 \mathrm{~h}$, incubated with primary antibodies (1:1,000 for anti-PRMT1, UPSTATE and 1:1,000 for anti-methylarginine antibodies ADMA from Cell Signaling, Danvers, MA, USA) overnight, washed three times in TTBS, then incubated with secondary antibody for $1 \mathrm{~h}$. Chemiluminescent detection using the VisGlow substrate for HRP (Visual Protein, Taipei City, Taiwan) was performed according to the manufacturer's instructions.

Cell growth assay. Aliquots of cultured cells were mixed with trypan blue and the cell numbers were counted using a hemocytometer. The MTT assay was used to evaluate cell proliferation. Cells seeded in 6-well plates $(10,000$ cells/well) were incubated with the MTT solution $(0.25 \mathrm{mg} / \mathrm{ml}$; SigmaAldrich, St. Louis, MO, USA) for $3 \mathrm{~h}$. The resulting formazan crystals were dissolved in dimethyl sulfoxide (DMSO) and measured by spectrophotometry at the absorbance of $570 \mathrm{~nm}$. 
Cell migration assay. Cells $\left(5 \times 10^{4}\right)$ were seeded into wells of the Oris Cell Migration Assembly Kit-FLEX (Platypus Technologies Llc, Fitchburg, WI, USA) and migration assays were conducted in accordance with the manufacturer's instructions. After attached for $10 \mathrm{~h}$, cells were allowed to migrate into the clear field with the removal of the well inserts. After migration for a period of time, cells were fixed with formaldehyde, stained with crystal violet and photographed. The pre-migration and post-migration images were analyzed using ImageJ software (http://rsb.info.nih.gov/ij/).

Stable shRNA-mediated PRMT1 knockdown in SAS cells. Lentiviral particles produced in packaging vector $\mathrm{pLKO}$ TRC005 with short hairpin RNA (shRNA) targeting human PRMT1 (A1 with the target sequences: 5'- GTGTTCCAGTAT CTCTGATTA-3'; B1 with the target sequences: 5'-CCGGC AGTACAAAGACTACAA-3') and a negative control were obtained from the National RNAi Core Facility (Academia Sinica, Taipei City, Taiwan). Cells were infected in complete growth medium supplemented with polybrene (SigmaAldrich). After $24 \mathrm{~h}$, cells were grown and selected in medium containing in $5 \mu \mathrm{g} / \mathrm{ml}$ puromycin (Sigma-Aldrich). The knockdown effects were examined by PRMT1 protein expression using immunoblotting.

Oral cancer specimens and immunohistochemical (IHC) studies. Paraffin embedded specimens (Pathological blocks) were from patients with head and neck cancers of different stages. The patients recruited in the Department of Otolaryngology, Chung-Shan Medical University Hospital (Taichung, Taiwan) between 2001 and 2010 were de-linked from their identification information and were randomly numbered. The present study was approved by the Institutional Research Board of Chung-Shan Medical University Hospital. IHC was conducted with the ultraView Universal DAB Detection kit (Roche) using the Ventana BenchMark XT automated IHC/ISH slide staining system (Roche) at the short $\mathrm{CC}$ antigen retrieval condition. Anti-PRMT1 antibodies from Upstate Biotechnologies were diluted (1:200) and incubated at $37^{\circ} \mathrm{C}$ for $16 \mathrm{~min}$.

\section{Results}

Protein arginine methyltransferase PRMT1 and methylarginine containing proteins in different oral cancer cell lines. We first examined protein arginine methylation in three oral squamous carcinoma cancer cell lines (SAS, OECM-1 and HSC-3). Immortalized S-G cells established from clinically normal gingiva was used as a normal control. In comparison, cell extracts prepared from other human cancer cell lines such as HeLa (cervical cancer cell line) and MCF-7 (breast cancer cell line) cells that have been investigated for PRMTs or arginine methylation (39-41) were included. The major type I protein arginine methyltransferase PRMT1 and ADMA-containing proteins were detected using specific antibodies. Western blot analyses showed that the expression patterns of PRMT1 in oral cancer cells were similar to that of HeLa and MCF-7 cells with the major signals of about the same molecular masses (Fig. 1A). The expression levels of PRMT1 was lowest in HSC-3.
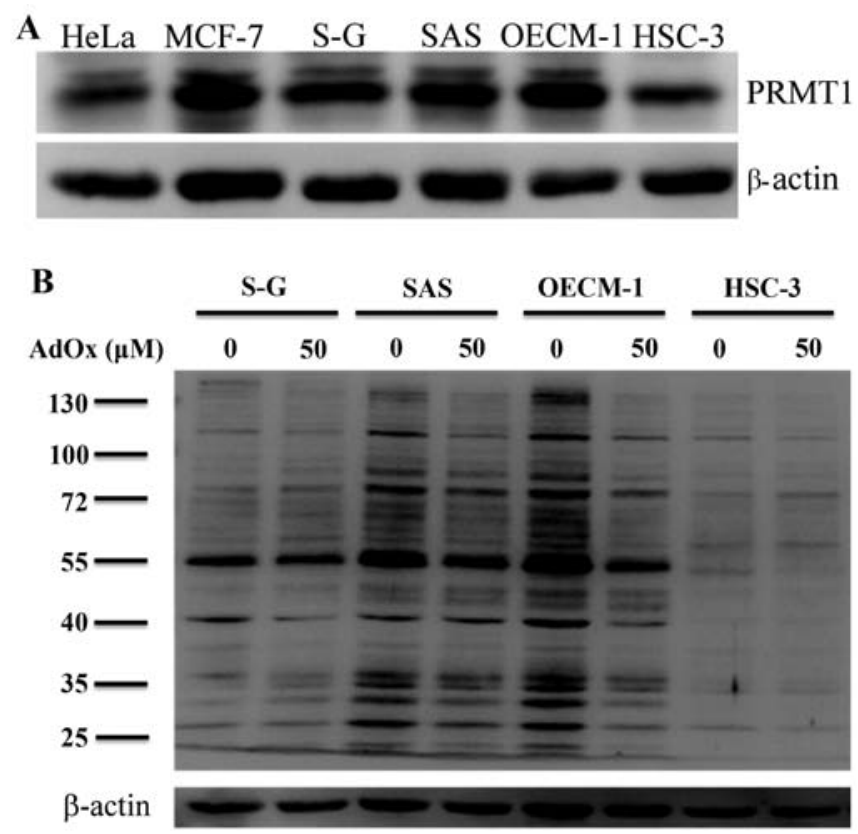

Figure 1. PRMT protein and arginine methylated protein expression in cancer cell lines. (A) Cell extract proteins $(15 \mu \mathrm{g})$ prepared from HeLa, MCF-7, S-G, SAS, OECM-1 and HSC-3 cells were analyzed by western blot analysis using anti-PRMT1 antibodies. (B) S-G, SAS, OECM-1 and HSC-3 cells were treated with $50 \mu \mathrm{M}$ AdOx or not for $24 \mathrm{~h}$, and the cell extracts were analyzed by western blot analysis using an asymmetric dimethyl-arginine (ADMA)specific antibody ADMA. Anti- $\beta$-actin was used as the loading control.

The levels of ADMA-containing proteins were high in OECM-1 and SAS, and lower in S-G cells (Fig. 1B). HSC-3 only showed few weak ADMA-containing signals. To analyze the effects of arginine methylation in the oral cancer cells, we treated the cells with an indirect methyltransferase inhibitor AdOx that has been widely used in protein methylation studies. AdOx treatment clearly decreased ADMA signals in oral cancer cell line SAS and OECM-1 that express high level of PRMT1, the major type I PRMT catalyzing the formation of ADMA (Fig. 1B; 50 mM AdOx). Decreased ADMA signals following the methylation inhibition by $\mathrm{AdOx}$ also confirmed that the signals detected by the ADMA-specific antibodies are from methylation modification. However, methylarginine levels reduced less significantly for HSC-3 and S-G cells.

Reduced growth rate of oral cancer cell lines, but not immortalized oral cells, after treatment with the methylation inhibitor AdOx. As a general transmethylation inhibitor, AdOx has been shown to block proliferation of different cancer cells $(33,36,37)$. To examine whether AdOx can affect growth of oral cancer cells, we first determined the growth curves of the four cell lines. SAS and OECM-1 proliferated at a higher growth rate compared to the S-G cells (Fig. 2A). On the other hand, HSC-3 cells that expressed low level of PRMT1 and ADMA-containing proteins, proliferated at a slower rate similar to the S-G cells. We then determined the effects of AdOx treatment on the proliferation of these cells. Addition of AdOx at the concentration that could effectively reduce the ADMA level $(50 \mathrm{mM})$ also blocked the proliferation of SAS and OECM-1 cells significantly (Fig. 2B). In contrast, cell 
Table I. HNC patients included in the PRMT1 IHC studies.

\begin{tabular}{|c|c|c|c|c|c|c|c|c|}
\hline Patient & Sex & Subside & $\begin{array}{c}\text { Age } \\
\text { (years) }\end{array}$ & $\begin{array}{l}\text { Tumor } \\
\text { T status }\end{array}$ & $\begin{array}{l}\text { Lymph node } \\
\text { metastasis }\end{array}$ & Metastasis & Stage & Cell differentiation \\
\hline 1 & Male & Tongue & 51 & 2 & 2 & 0 & IVA & Poorly differentiated \\
\hline 2 & Male & Tongue & 55 & 1 & 2 & 0 & IVA & Moderately differentiated \\
\hline 3 & Female & Buccal mucosa & 72 & 1 & 0 & 0 & I & Moderately differentiated \\
\hline 4 & Male & Buccal mucosa & 57 & 1 & 0 & 0 & I & Well differentiated \\
\hline 5 & Male & Buccal mucosa & 71 & 1 & 0 & 0 & I & Verrucous carcinoma \\
\hline 6 & Female & Tongue & 52 & 1 & 0 & 0 & I & Poorly differentiated \\
\hline
\end{tabular}

A
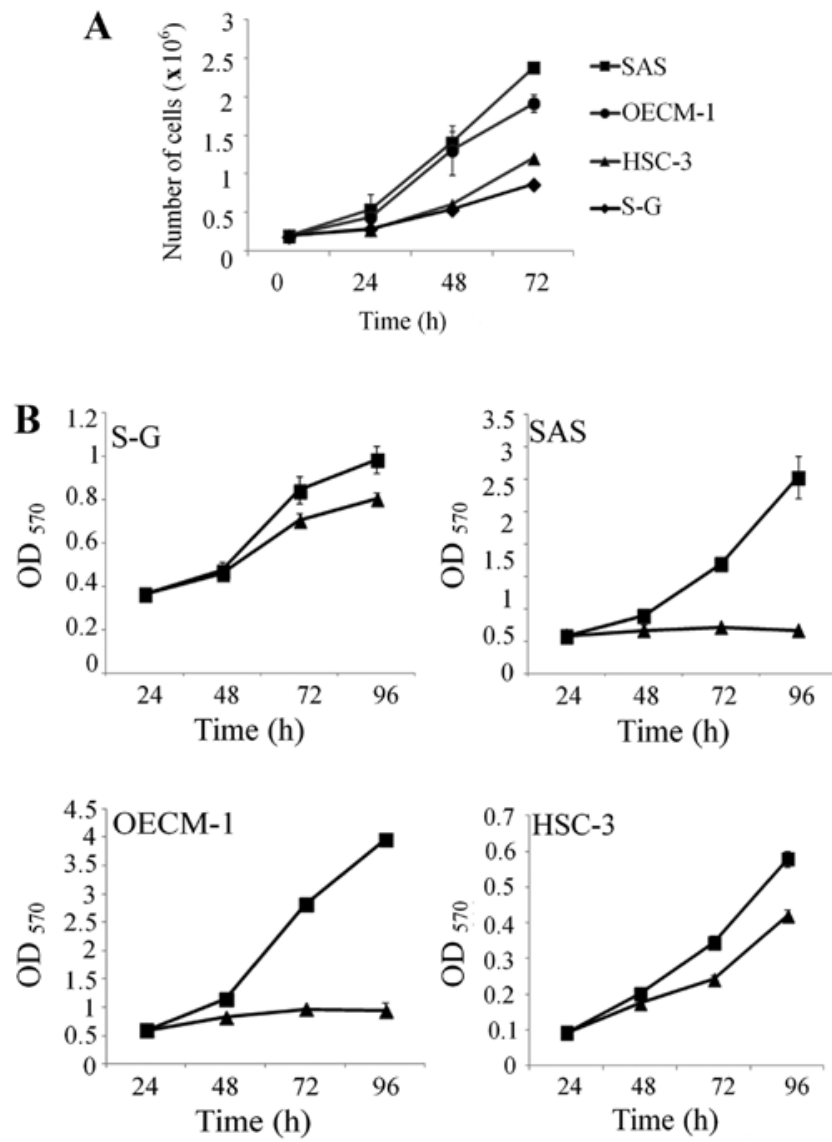

Figure 2. The growth rate of oral cancer cells and the effects of AdOx treatment on cell proliferation. (A) The growth curves of S-G, SAS, OECM-1 and HSC-3 cells determined by direct cell count using a hemocytometer at 24,48 and 72 h. (B) S-G, SAS, OECM-1 and HSC-3 cells were treated with AdOx for $24 \mathrm{~h}$ and cell proliferation was analyzed by MTT assay. The triangles and the squares indicated the number of AdOx treated and untreated cells, respectively.

growth of the S-G cells was only slightly inhibited by the same AdOx treatment. Limited growth inhibition of the HSC-3 cells by AdOx treatment was observed (Fig. 2B).

Migration activity of SAS oral cancer cells decreases after AdOx treatment. AdOx treatment has also been demonstrated to suppress tumor cell invasion and migration (35). We thus evaluated the effects of $\mathrm{AdOx}$ on migration of the oral cancer cells. Analyses of the migration activity of the oral cancer cells showed migration activity of SAS and HSC-3 but limited migration ability of OECM-1 (data not shown). We thus used SAS and HSC-3 cells to study the effects of AdOx on migration. It is apparent that HSC-3 migrated faster than SAS (Fig. 3A, $16 \mathrm{~h}$ ). Treatment with $50 \mathrm{mM}$ of AdOx reduced the migration of SAS and HSC-3 cells (Fig. 3A). To analyze the effects of $\mathrm{AdOx}$ treatment on the migration activity of the cells, migration area of the AdOx-treated cells was compared with that of the untreated ones. After the treatment, the area covered by the migrated SAS cells was only about $30 \%$ of the area covered by the migrated untreated cells. The migration activity of HSC-3 cells was slightly suppressed with the migration area close to $70 \%$ of that of untreated cells (Fig. 3B).

Elevated expression of PRMT1 in oral cancer specimens. To further investigate the clinical involvement of PRMT1 in HNC, we analyzed PRMT1 expression in oral cancer specimens by immunohistochemistry. Six patients were included in the present study as described in Table I. We included tissue sections from patients of the late (IVA) or early (I) oral cavity SCC stages and the subside from tongue or buccal mucosa. Representative IHC stains of all six patients are shown in Fig. 4A. To better illustrate the PRMT1 expression in tumor and normal cells, representative regions containing tumor or normal cells from patients 4, 5 and 6 are shown in Fig. 4B with higher magnification. It is clear that PRMT1 stained weakly in the normal cells but its expression were stronger in the poorly differentiated tumor cells. The majority of the intense PRMT1 signals were concentrated in the nucleus. As illustrated in patients 4-6, the IHC stain of PRMT1 revealed strong nuclear staining in cancer cells (upper panels) and weak nuclear staining in non-neoplastic squamous cells (lower panels). Even though the samples were limited in the present study, the expression level of PRMT1 in the specimens appeared to have strong correlation with the tumors. The PRMT1 level did not appear to be correlated with the cancer stage, subside or sex.

We also analyzed the head and neck squamous carcinoma database from The Cancer Genome Atlas (TCGA; 273 samples) by SurvExpress (http://bioinformatica.mty. itesm.mx/SurvExpress) (42). In SurvExpress, all datasets from TCGA were obtained at the gene level (level 3) which were the data of RNA-Seq (42). It is apparent that the highrisk group express higher level of PRMT1 than the low-risk group (Fig. 4C). Similar results can be obtained from another database (TCGA Head and Neck squamous cell carcinoma June 2016, 502 samples). Furthermore, extended survival of 
A
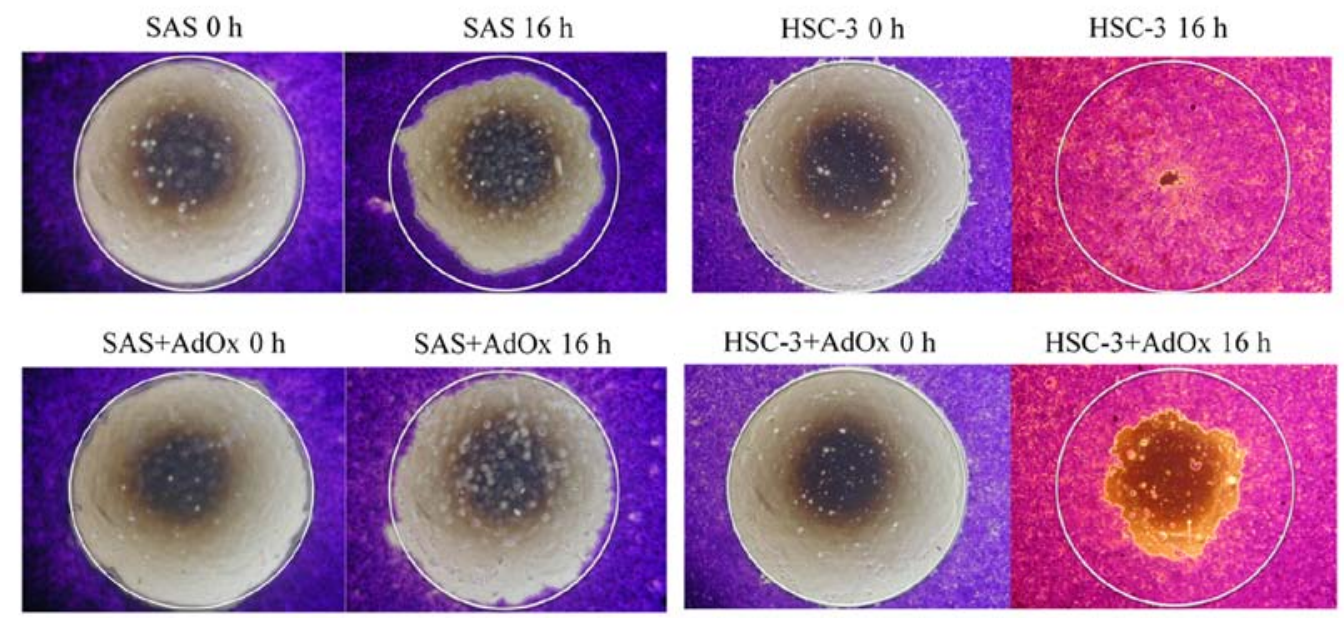

\section{B}
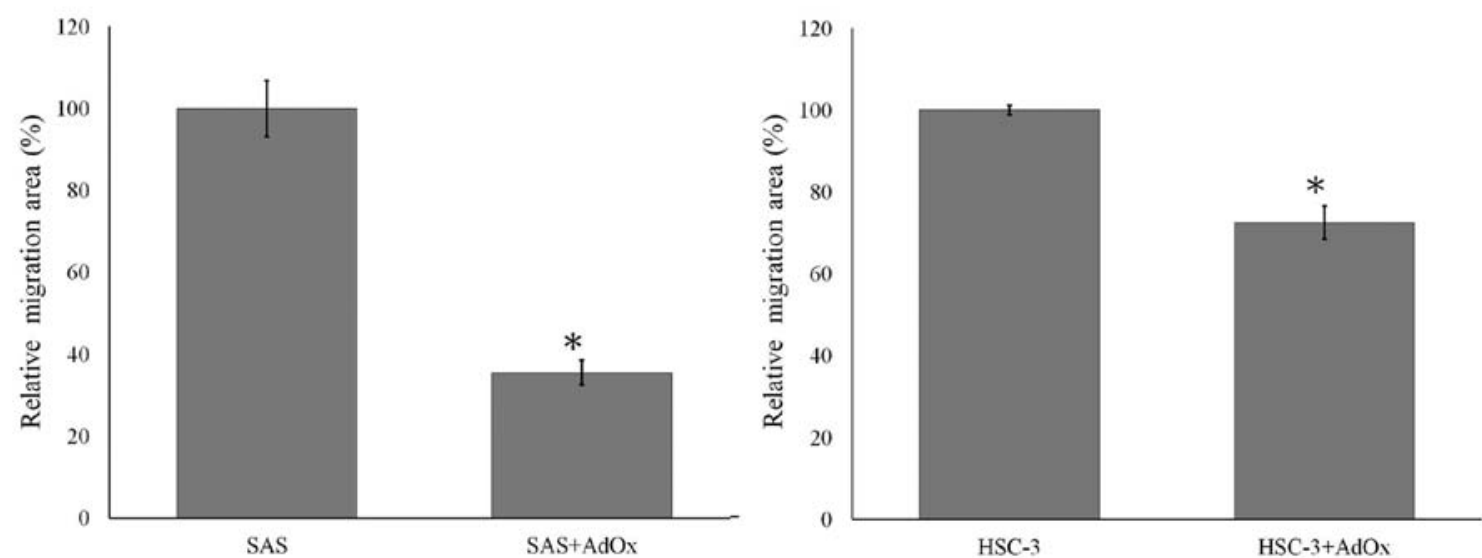

Figure 3. Reduced migration of SAS and HSC-3 cells after AdOx treatment. (A) Images of SAS and HSC-3 cells stained with crystal violet pre-migration $(0 \mathrm{~h})$ and post-migration $(16 \mathrm{~h})$ are shown. After the cells had attached to the plates, the stoppers in the center were removed. The white circles indicate areas covered by the stoppers before cell migration. Within the doubling time of the cells, the covering of the clear field was considered to be due to cell migration. The area inside the white circle covered by cells is the migration area. (B) Quantification of cell movement was represented as the percentage of the migration area covered by migrated cells of AdOx treated cells compared to that of the untreated control cells. Relative migration area is shown as the mean \pm SD of two independent experiments performed in quadruplicate. A statistically significant difference between the AdOx-treated and control cells is indicated ( $\mathrm{P}<0.01$; Student's t-test).

the PRMT1 low expression group is statistically significant $(\mathrm{P}<0.05)$ compared with the high expression ones in either the stage II (Fig. 4D) or grade II samples (data not shown).

Knockdown of PRMT1 in SAS oral cancer cells decreases proliferation and migration. As arginine methylation stands for the majority of the AdOx-blocked protein methylation (38) and PRMT1 is the predominant type I PRMT, AdOx might affect mostly through PRMT1 for decreased migration activity. Furthermore, PRMT1 expression was strongest in the poorly differentiated cells in the clinical samples. We thus suspect that PRMT1 is critical for the proliferation and migration/invasion activity of HNC. To specifically analyze the function of PRMT1 in oral cancer cells, we knocked down PRMT1 gene in SAS cells with shRNA by lentivirus (shPRMT1A1, B1 or combined) infection. The protein level of PRMT1 decreased almost completely in cells that stably express either or both of the two different PRMT1 shRNA (PRMT1-KD-1, PRMT1-KD-2 or PRMT1-KD) compared to that in the non-infected or control shRNA infected SAS cells
(SAS and Control; Fig. 5A). As PRMT1 is the major type I PRMT, the level of proteins containing asymmetric dimethylarginine (ADMA) also decreased significantly after the PRMT1 knockdown (data not shown). We then examined the growth of SAS cells with PRMT1 knockdown. The PRMT1 KD cells apparently grew slower than the non-infected or control shRNA infected cells. Treatment of the cells with AdOx further reduced the growth of all three cells with or without PRMT1 KD (Fig. 5B).

We also determined the migration abilities of the SAS cells. The migration of the SAS cells with PRMT1 knockdown showed significantly decreased migration compared with the cells with control shRNA or SAS cells (Fig. 5C and D). E-cadherin is a calcium-dependent adhesion molecule expressed on normal epithelium (43). The elevated expression of E-cadherin has been reported to suppress cell migration (44). We further examined the expression of E-cadherin in PRMT1 knockdown SAS cells and the results showed that PRMT1 knockdown upregulated the epithelial marker E-cadherin (Fig. 5A). 
$\mathbf{A}$

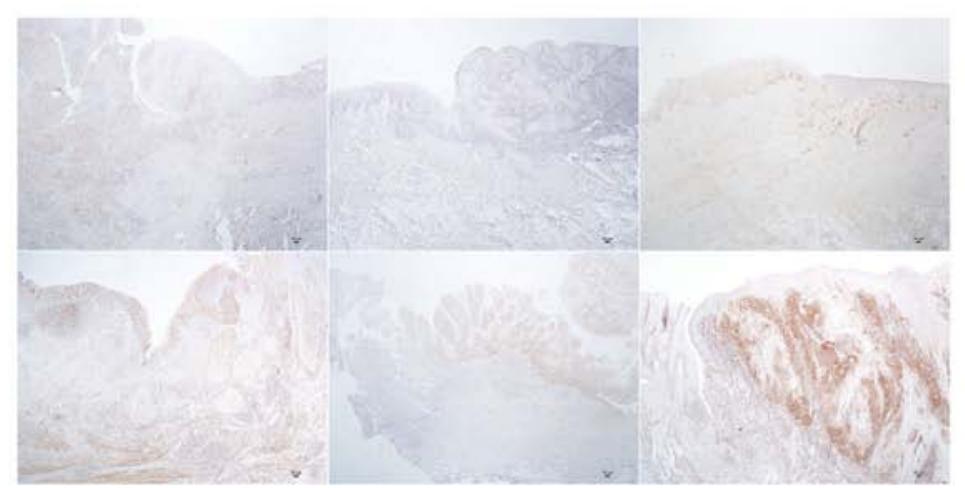

\begin{tabular}{l|l|l}
$\mathrm{pt} 1$ & $\mathrm{pt} 2$ & $\mathrm{pt} 3$ \\
\hline $\mathrm{pt} 4$ & $\mathrm{pt} 5$ & $\mathrm{pt} 6$
\end{tabular}

B

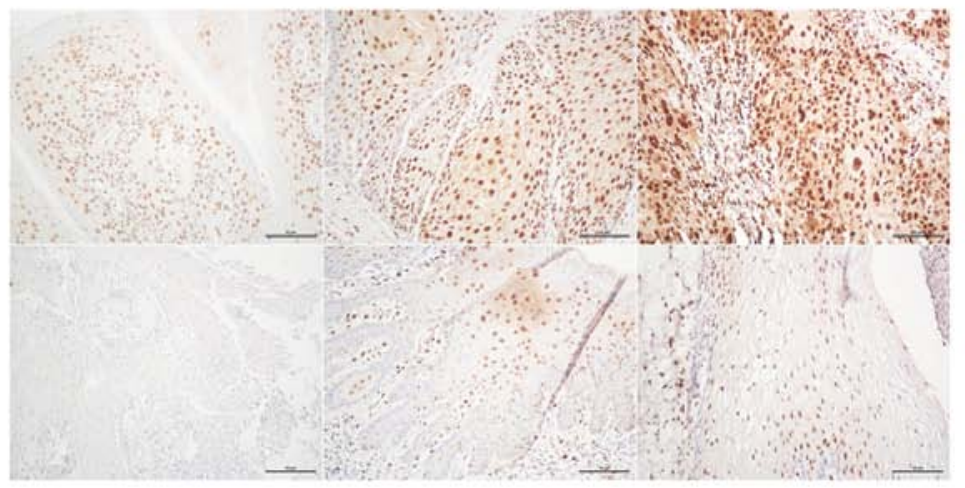

\begin{tabular}{l|l|l|l}
$4 \mathrm{~T}$ & $5 \mathrm{~T}$ & $6 \mathrm{~T}$ \\
\hline $4 \mathrm{~N}$ & $5 \mathrm{~N}$ & $6 \mathrm{~N}$
\end{tabular}

C

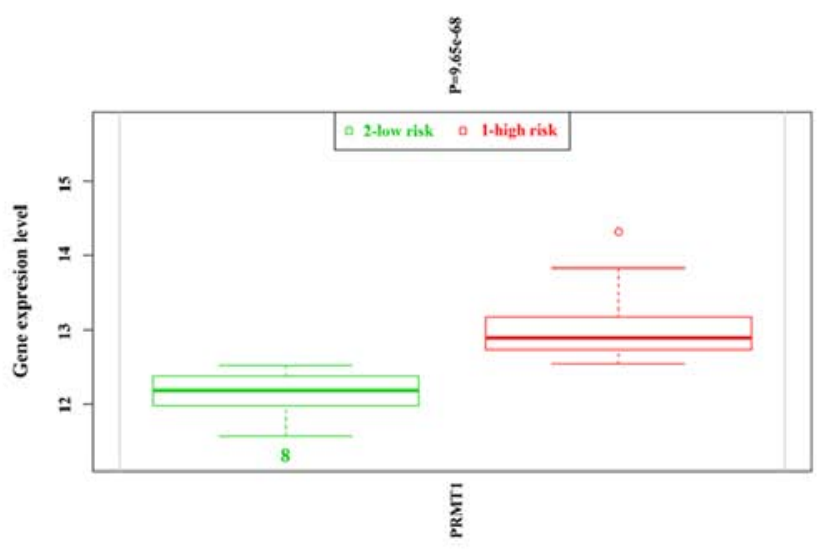

D

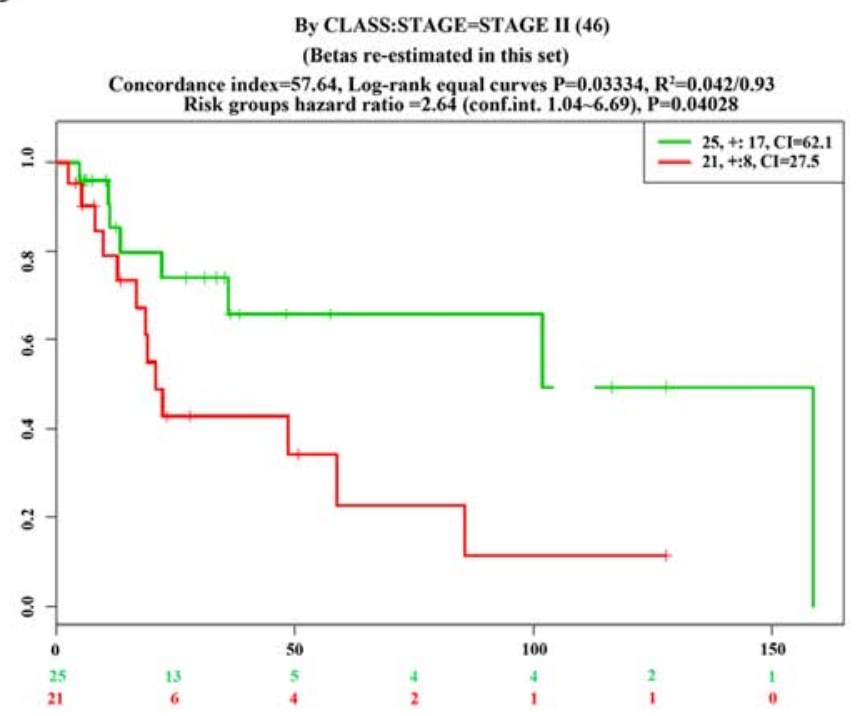

Figure 4. Increased PRMT1 expression in tumor cells in oral cancer specimens. Tissue specimens from six different HNC patients were stained with antiPRMT1 antibodies. (A) Magnification, x20. IHC of tissue sections from patient 1 to patient 6 are shown as depicted in the right panel. (B) Magnification, x200. The regions mostly with tumor and with non-tumor cells from sections of patients 4, 5 and 6 are shown in the upper and lower panels. (C) Correlation of PRMT1 expression level and survival of head and neck cancer patients. Expression levels of PRMT1 in high-risk and low-risk groups were analyzed using head and neck squamous carcinoma database from The Cancer Genome Atlas (TCGA; 273 samples) by SurvExpress (http://bioinformatica.mty.itesm.mx/ SurvExpress). High-risk or low-risk groups were divided according to the Prognostic Index (Risk score) of the patients. (D) Extended survival of the PRMT1 low expression group is statistically significant $(\mathrm{P}<0.05)$ compared with the high expression ones in the stage II samples.

\section{Discussion}

Besides as players of epigenetic modifiers, protein arginine methyltransferases are known to methylate a plethora of substrates involved in transcriptional regulation, signal transduction and DNA methylation. The involvement of protein arginine methylation or PRMTs has been analyzed in different cancers but not in head and neck cancer. We thus started the investigation using oral cancer cells.

We observed that two oral cancer cell lines SAS and OECM-1 expressed high ADMA level while another oral cancer cell line HSC-3 contained low level of ADMA- 
A

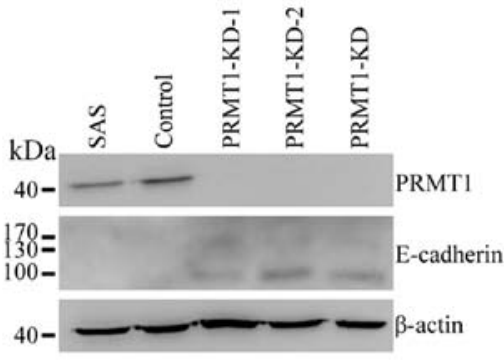

B

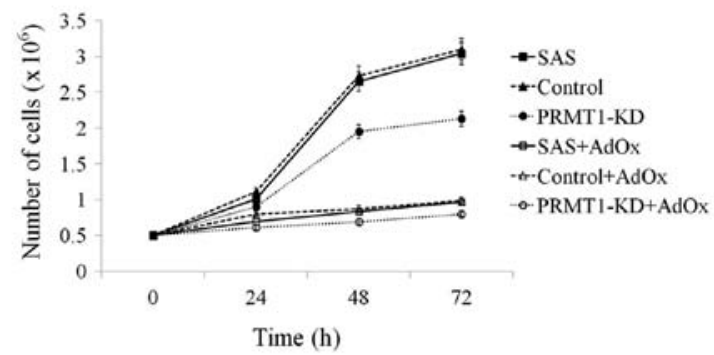

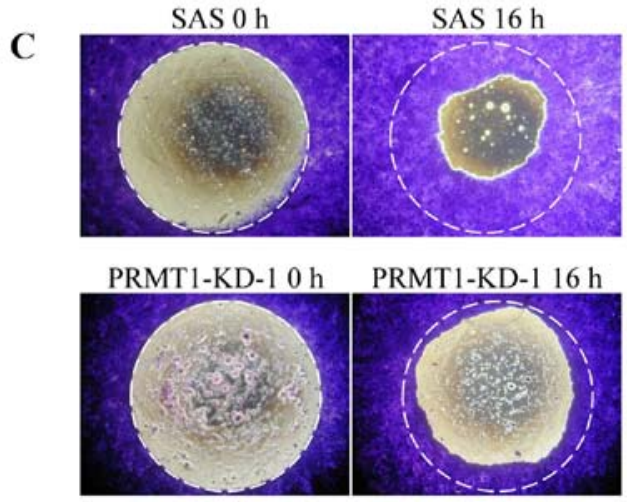

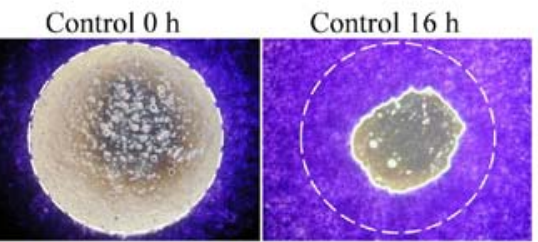

PRMTI-KD-2 $0 \mathrm{~h}$

PRMT1-KD-2 $16 \mathrm{~h}$
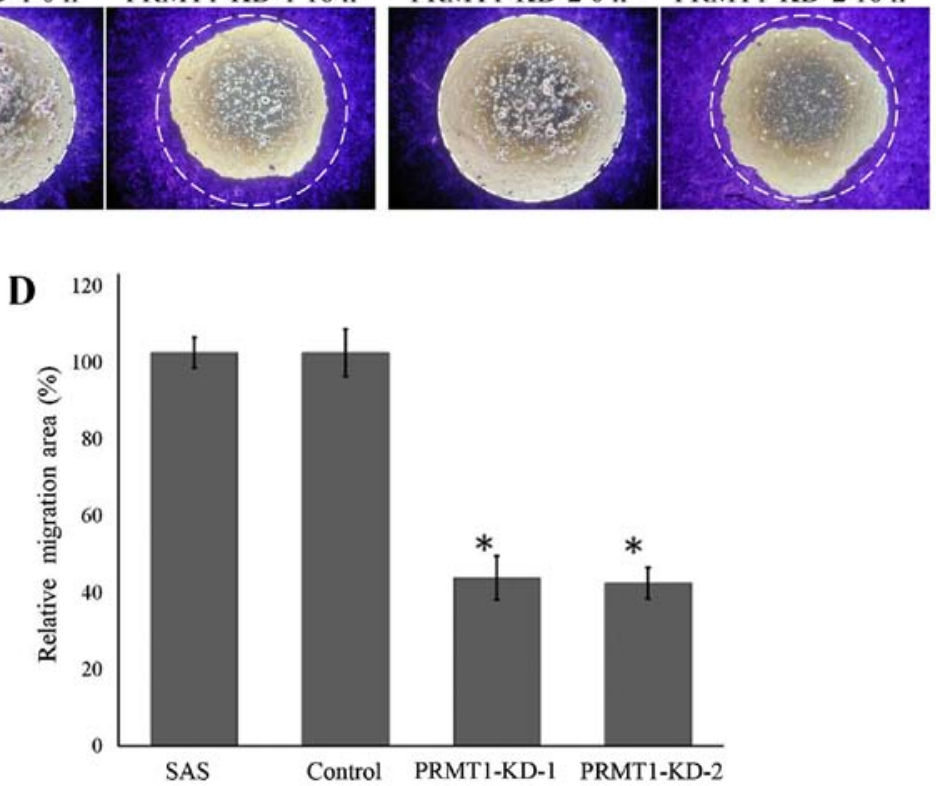

Figure 5. Knockdown of PRMT1 in SAS oral cancer cells decreased migration activity. (A) PRMT1 in SAS oral cancer cells can be effectively knocked down by lentivirus shPRMT1 infection as shown by western blot analyses. Cell extracts prepared from the SAS cells not infected, infected with shCtrl (control), shPRMT1A1 (PRMT1-KD-1), B1 (PRMT1-KD-2) or both shPRMT1 (PRMT1-KD) were detected with anti-PRMT1 antibodies. Expression of E-cadherin in the same SAS cell extract samples were detected by western blot analyses. Anti- $\beta$-actin was detected as the loading control. (B) The growth curves of SAS, shCtrl infected SAS (control) or shPRMT1 infected (PRMT1-KD) SAS cells treated or not treated with AdOx (50 mM) were determined by direct cell count using a hemocytometer at 24, 48 and $72 \mathrm{~h}$. (C) Images of SAS cells not infected, infected with shCtrl (control) or shPRMT1 (PRMT1-KD-1 and PRMT1-KD-2) pre-migration $(0 \mathrm{~h})$ and post-migration $(16 \mathrm{~h})$ are shown. The white circles indicate areas covered by the stoppers before cell migration. The area inside the white circle covered by cells is the migration area. (D) Quantification of migration activity of the cells were shown as the percentage of the migration area of shCtrl or shPRMT1 infected cells compared to that of uninfected SAS cells ("P<0.01; Student's t-test).

containing proteins. Consistently, the expression level of the major type I protein arginine methyltransferase PRMT1 is high in SAS and OECM-1 but low in HSC-3 cells. The expression level of PRMT1 and ADMA-containing proteins in HSC-3 cells were even lower than that in the immortalized normal S-G cells. The results might reflect the heterogeneous nature of oral cancer formation and suggests that oral cancer cell lines expressing different levels of PRMTs might have diverse PRMT involvement in their carcinogenesis.

The indirect methyltransferase inhibitor AdOx has been shown to decrease cancer cell survival and induce apoptosis $(33,36,37)$. SAS and OECM-1, the two oral cancer cells with high level of ADMA-containing polypeptides, showed more significant decrease of the ADMA level than the S-G and HSC-3 cells when treated with AdOx. Treatment of AdOx at the concentration $(50 \mathrm{mM})$ that could effectively reduce ADMA levels in SAS and OECM1 oral cancer cells also inhibited their cell growth. The same AdOx treatment that reduced less ADMA level in S-G and HSC-3 cells also only decreased the growth rate slightly. It is likely that cancer cells with high PRMT1/ADMA levels have a higher proliferation rate and $\mathrm{AdOx}$ treatment can reduce their growth more significantly than the ones with low ADMA levels.

We also showed that AdOx treatment can reduce the migration activity of the oral cancer cells SAS and HSC-3. In comparison, migration of SAS can be inhibited to a greater 
extent than that of the HSC-3 cells. AdOx treatment can effectively block cell proliferation in SAS and OECM1 and inhibit migration in SAS and HSC-3. Thus, AdOx can have the potential to reduce growth or migration of the oral cancer cells depending on PRMT1/ADMA levels and the nature of the cells.

We suspect that inhibition of PRMT1 might be responsible for most of the AdOx effects. Besides as an epigenetic modifier, PRMT1 is known to methylate a plethora of substrates involved in transcriptional regulation, signal transduction and DNA methylation. Indeed knockdown of PRMT1 phenocopied the effects of AdOx treatment on SAS cells. Furthermore, PRMT1 has been shown to be highly expressed in various cancers including bladder (17), liver (45) and esophageal cancer (46). We showed overexpression of PRMT1 in the tumor cells compared with the neighboring normal cells in tissue sections from head and neck cancer patients. The PRMT1 staining is highly concentrated in the nucleus and is thus likely to play roles in transcriptional regulation. Even though the tissue samples were limited, increased high PRMT1 expression level appeared to be typical in the tumor cells. We included stage I and stage IV patients for IHC stains but the expression levels of PRMT1 in the specimens were not correlated with the cancer stage. Nevertheless, HNSCC data from TCGA confirmed the high expression of PRMT1 in the high-risk group and extended survival of the low PRMT1 expression patients especially of grade II or stage II.

We knocked down the expression of PRMT1 in the SAS cells that express high level of PRMT1 and show high migration activity. After knockdown of PRMT1 in SAS, we observed reduced mobility of the cells. PRMT1 has been shown to induce epithelial-mesenchymal transition (EMT) in lung cancer cells (47) and breast cancer cells (48). Upregulated PRMT1 catalyzed the methylation of Twist1 for E-cadherin repression in lung cancer cell lines (47). PRMT1 also activated the transcription of another E-cadherin repressor ZEB1 in breast cancer cells through epigenetic regulation (48). Increased expression of the epithelial marker E-cadherin after PRMT1 knockdown in SAS cells was detected. The mechanism of the inhibitory effect of PRMT1 KD to the mobility of SAS cells requires further investigation.

Recently methylation of epidermal growth factor receptor (EGFR) by PRMT1 has been shown to be related to the activation of the EGF signaling and might resulted in resistance to cetuximab, a targeted therapeutic monoclonal antibody to EGFR. Colon cancer as well as HNC patients with higher levels of methyl-EGFR in tumors have been shown to have a higher recurrence rate after cetuximab treatment (11). A gastric cancer study showed that low PRMT1 and thus low-nuclear FOXO1 levels were associated with higher recurrence after adjuvant chemotherapy and poor prognosis (49). Furthermore, they showed that the reduction in PRMT1 expression in gastric cancer cell lines significantly inhibited sensitivity to cisplatin and 5-fluorouracil (49). Chemotherapy with both agents is also the standard treatment of choice for HNSCC. The effects of PRMT1 on these agents can be evaluated and as PRMT1 expression is heterogeneous in HNC patients and oral cancer cell lines, it should be helpful to analyze the PRMT1 level for proper treatment.
Though elevated PRMT1 expression in various cancer has been suggested to function in tumorigenesis, PRMT1 might still play some roles in tumor suppression. For example, PRMT1 was shown to have ordered cooperative roles with p300 and CARM1 as a coactivator for the master tumor suppressor p53 (50). Moreover, repression of the $\mathrm{NF}-\kappa \mathrm{B}$ pathway critical for inflammation and cancer by PRMT1 through arginine methylation of the RelA subunit was reported recently (51). The effects of PRMT1 on specific cancer might have to consider the major signals in conjunction with the microenvironments leading to the tumor formation. The heterogeneous nature of oral cancers and the origin of the cancer cell lines might explain the variable levels of ADMA and PRMT1 in different oral cancer cells in this study. Interestingly, the efficiency of a methylation inhibitor AdOx to inhibit cell proliferation or migration was correlated with the level of ADMA-containing protein and the type I PRMT proteins in the oral cancer cells. Thus, while AdOx treatment can greatly inhibit growth and migration of SAS, it worked limitedly with HSC-3 in comparison. Further application of methylation inhibition in therapeutic use should require analyses of PRMT expression and ADMA levels on pathological oral cancer specimens. The present study thus provides fundamental background for future evaluation of the PRMT genes as the therapeutic targets of oral cancer as well as using AdOx or related compounds for oral cancer treatment.

\section{Acknowledgements}

The present study was supported by the CSH-2014-C-021 from the Chung Shan Medical University Hospital to C.C. and MOST 103-2320-B-040-022-MY3 from the Ministry of Science and Technology to C.L. We thank Professor Shun-Fa Yang for providing the HSC-3 cells.

\section{References}

1. Chen QW, Zhu XY, Li YY and Meng ZQ: Epigenetic regulation and cancer (Review). Oncol Rep 31: 523-532, 2014.

2. Yang Y and Bedford MT: Protein arginine methyltransferases and cancer. Nat Rev Cancer 13: 37-50, 2012.

3. Bedford MT and Clarke SG: Protein arginine methylation in mammals: Who, what, and why. Mol Cell 33: 1-13, 2009.

4. Krause CD, Yang ZH, Kim YS, Lee JH, Cook JR and Pestka S: Protein arginine methyltransferases: Evolution and assessment of their pharmacological and therapeutic potential. Pharmacol Ther 113: 50-87, 2007

5. Wang YC and Li C: Evolutionarily conserved protein arginine methyltransferases in non-mammalian animal systems. FEBS J 279: 932-945, 2012.

6. Yang Y, Hadjikyriacou A, Xia Z, Gayatri S, Kim D, ZuritaLopez C, Kelly R, Guo A, Li W, Clarke SG, et al: PRMT9 is a type II methyltransferase that methylates the splicing factor SAP145. Nat Commun 6: 6428, 2015.

7. Zurita-Lopez CI, Sandberg T, Kelly R and Clarke SG: Human protein arginine methyltransferase 7 (PRMT7) is a type III enzyme forming $\omega$-NG-monomethylated arginine residues. J Biol Chem 287: 7859-7870, 2012.

8. Jansson M, Durant ST, Cho EC, Sheahan S, Edelmann M, Kessler B and La Thangue NB: Arginine methylation regulates the p53 response. Nat Cell Biol 10: 1431-1439, 2008.

9. Le Romancer M, Treilleux I, Leconte N, Robin-Lespinasse Y, Sentis S, Bouchekioua-Bouzaghou K, Goddard S, GobertGosse $S$ and Corbo L: Regulation of estrogen rapid signaling through arginine methylation by PRMT1. Mol Cell 31: 212-221, 2008 . 
10. Guendel I, Carpio L, Pedati C, Schwartz A, Teal C, Kashanchi F and Kehn-Hall K: Methylation of the tumor suppressor protein, BRCA1, influences its transcriptional cofactor function. PLoS One 5: e11379, 2010

11. Liao HW, Hsu JM, Xia W, Wang HL, Wang YN, Chang WC Arold ST, Chou CK, Tsou PH, Yamaguchi H, et al: PRMT1mediated methylation of the EGF receptor regulates signaling and cetuximab response. J Clin Invest 125: 4529-4543, 2015.

12. Auclair Y and Richard S: The role of arginine methylation in the DNA damage response. DNA Repair (Amst) 12: 459-465, 2013.

13. Bikkavilli RK, Avasarala S, Vanscoyk M, Sechler M, Kelley N, Malbon CC and Winn RA: Dishevelled3 is a novel arginine methyl transferase substrate. Sci Rep 2: 805, 2012.

14. Bikkavilli RK and Malbon CC: Arginine methylation of G3BP1 in response to Wnt3a regulates $\beta$-catenin mRNA. J Cell Sci 124: 2310-2320, 2011.

15. Blythe SA, Cha SW, Tadjuidje E, Heasman J and Klein PS: betaCatenin primes organizer gene expression by recruiting a histone H3 arginine 8 methyltransferase, Prmt2. Dev Cell 19: 220-231, 2010.

16. Cha B, Kim W, Kim YK, Hwang BN, Park SY, Yoon JW, Park WS, Cho JW, Bedford MT and Jho EH: Methylation by protein arginine methyltransferase 1 increases stability of Axin, a negative regulator of Wnt signaling. Oncogene 30: 2379-2389, 2011.

17. Yoshimatsu M, Toyokawa G, Hayami S, Unoki M, Tsunoda T, Field HI, Kelly JD, Neal DE, Maehara Y, Ponder BA, et al: Dysregulation of PRMT1 and PRMT6, Type I arginine methyltransferases, is involved in various types of human cancers. Int J Cancer 128: 562-573, 2011.

18. Limm K, Ott C, Wallner S, Mueller DW, Oefner P, Hellerbrand C and Bosserhoff AK: Deregulation of protein methylation in melanoma. Eur J Cancer 49: 1305-1313, 2013.

19. Al-Dhaheri M, Wu J, Skliris GP, Li J, Higashimato K, Wang Y, White KP, Lambert P, Zhu Y, Murphy L, et al: CARM1 is an important determinant of ER $\alpha$-dependent breast cancer cell differentiation and proliferation in breast cancer cells. Cancer Res 71: 2118-2128, 2011.

20. Baldwin RM, Morettin A, Paris G, Goulet I and Côté J: Alternatively spliced protein arginine methyltransferase 1 isoform PRMT1v2 promotes the survival and invasiveness of breast cancer cells. Cell Cycle 11: 4597-4612, 2012.

21. Dowhan DH, Harrison MJ, Eriksson NA, Bailey P, Pearen MA, Fuller PJ, Funder JW, Simpson ER, Leedman PJ, Tilley WD, et al: Protein arginine methyltransferase 6-dependent gene expression and splicing: Association with breast cancer outcomes. Endocr Relat Cancer 19: 509-526, 2012.

22. Goulet I, Gauvin G, Boisvenue S and Côté J: Alternative splicing yields protein arginine methyltransferase 1 isoforms with distinct activity, substrate specificity, and subcellular localization. J Biol Chem 282: 33009-33021, 2007.

23. Mathioudaki K, Scorilas A, Ardavanis A, Lymberi P, Tsiambas E, Devetzi M, Apostolaki A and Talieri M: Clinical evaluation of PRMT1 gene expression in breast cancer. Tumour Biol 32 575-582, 2011

24. Kim YR, Lee BK, Park RY, Nguyen NT, Bae JA, Kwon DD and Jung C: Differential CARM1 expression in prostate and colorectal cancers. BMC Cancer 10: 197, 2010.

25. Majumder S, Liu Y, Ford OH III, Mohler JL and Whang YE: Involvement of arginine methyltransferase CARM1 in androgen receptor function and prostate cancer cell viability. Prostate 66: 1292-1301, 2006.

26. Zakrzewicz D, Zakrzewicz A, Preissner KT, Markart P and Wygrecka M: Protein arginine methyltransferases (PRMTs) Promising targets for the treatment of pulmonary disorders. Int J Mol Sci 13: 12383-12400, 2012.

27. Mathioudaki K, Papadokostopoulou A, Scorilas A, Xynopoulos D, Agnanti $\mathrm{N}$ and Talieri M: The PRMT1 gene expression pattern in colon cancer. Br J Cancer 99: 2094-2099, 2008.

28. Papadokostopoulou A, Mathioudaki K, Scorilas A, Xynopoulos D, Ardavanis A, Kouroumalis E and Talieri M: Colon cancer and protein arginine methyltransferase 1 gene expression. Anticancer Res 29: 1361-1366, 2009.

29. Park BJ, Chiosea SI and Grandis JR: Molecular changes in the multistage pathogenesis of head and neck cancer. Cancer Biomark 9: 325-339, 2010.

30. Tang J, Frankel A, Cook RJ, Kim S, Paik WK, Williams KR, Clarke S and Herschman HR: PRMT1 is the predominant type I protein arginine methyltransferase in mammalian cells. J Biol Chem 275: 7723-7730, 2000
31. Najbauer J and Aswad DW: Diversity of methyl acceptor proteins in rat pheochromocytoma (PC12) cells revealed after treatment with adenosine dialdehyde. J Biol Chem 265: 12717-12721, 1990.

32. Najbauer J, Johnson BA and Aswad DW: Analysis of stable protein methylation in cultured cells. Arch Biochem Biophys 293: 85-92, 1992.

33. Hong S, Heo J, Lee S, Heo S, Kim SS, Lee YD, Kwon M and Hong S: Methyltransferase-inhibition interferes with neuronal differentiation of P19 embryonal carcinoma cells. Biochem Biophys Res Commun 377: 935-940, 2008.

34. Yan L, Zhao HY, Zhang Y and Shen YF: Differential effects of AdOx on gene expression in P19 embryonal carcinoma cells. BMC Neurosci 13: 6, 2012

35. Kim JH, Kim JH, Kim SC, Yi YS, Yang WS, Yang Y, Kim HG, Lee JY, Kim KH, Yoo BC, et al: Adenosine dialdehyde suppresses MMP-9-mediated invasion of cancer cells by blocking the Ras/ Raf-1/ERK/AP-1 signaling pathway. Biochem Pharmacol 86: $1285-1300,2013$

36. Kim JH, Lee YG, Yoo S, Oh J, Jeong D, Song WK, Yoo BC, Rhee MH, Park J, Cha SH, et al: Involvement of Src and the actin cytoskeleton in the antitumorigenic action of adenosine dialdehyde. Biochem Pharmacol 85: 1042-1056, 2013.

37. Schwerk $C$ and Schulze-Osthoff K: Methyltransferase inhibition induces p53-dependent apoptosis and a novel form of cell death. Oncogene 24: 7002-7011, 2005

38. Li C, Ai LS, Lin CH, Hsieh M, Li YC and Li SY: Protein $\mathrm{N}$-arginine methylation in adenosine dialdehyde-treated lymphoblastoid cells. Arch Biochem Biophys 351: 53-59, 1998.

39. Chen DH, Wu KT, Hung CJ, Hsieh M and Li C: Effects of adenosine dialdehyde treatment on in vitro and in vivo stable protein methylation in HeLa cells. J Biochem 136: 371-376, 2004.

40. Hung CJ, Lee YJ, Chen DH and Li C: Proteomic analysis of methylarginine-containing proteins in HeLa cells by twodimensional gel electrophoresis and immunoblotting with a methylarginine-specific antibody. Protein J 28: 139-147, 2009.

41. Herrmann F, Pably P, Eckerich C, Bedford MT and Fackelmayer FO: Human protein arginine methyltransferases in vivo - distinct properties of eight canonical members of the PRMT family. J Cell Sci 122: 667-677, 2009.

42. Aguirre-Gamboa R, Gomez-Rueda H, Martínez-Ledesma E, Martínez-Torteya A, Chacolla-Huaringa R, RodriguezBarrientos A, Tamez-Peña JG and Treviño V: SurvExpress: An online biomarker validation tool and database for cancer gene expression data using survival analysis. PLoS One 8: e74250, 2013.

43. van Roy F and Berx G: The cell-cell adhesion molecule E-cadherin. Cell Mol Life Sci 65: 3756-3788, 2008.

44. Perl AK, Wilgenbus P, Dahl U, Semb H and Christofori G: A causal role for E-cadherin in the transition from adenoma to carcinoma. Nature 392: 190-193, 1998.

45. Li B, Liu L, Li X and Wu L: miR-503 suppresses metastasis of hepatocellular carcinoma cell by targeting PRMT1. Biochem Biophys Res Commun 464: 982-987, 2015.

46. Zhou W, Yue H, Li C, Chen H and Yuan Y: Protein arginine methyltransferase 1 promoted the growth and migration of cancer cells in esophageal squamous cell carcinoma. Tumour Biol 37: 2613-2619, 2016.

47. Avasarala S, Van Scoyk M, Karuppusamy Rathinam MK, Zerayesus S, Zhao X, Zhang W, Pergande MR, Borgia JA, DeGregori J, Port JD, et al: PRMT1 is a novel regulator of epithelial-mesenchymal-transition in non-small cell lung cancer. J Biol Chem 290: 13479-13489, 2015.

48. Gao Y, Zhao Y, Zhang J, Lu Y, Liu X, Geng P, Huang B, Zhang Y and Lu J: The dual function of PRMT1 in modulating epithelialmesenchymal transition and cellular senescence in breast cancer cells through regulation of ZEB1. Sci Rep 6: 19874, 2016.

49. Altan B, Yokobori T, Ide M, Mochiki E, Toyomasu Y, Kogure N, Kimura A, Hara K, Bai T, Bao P, et al: Nuclear PRMT1 expression is associated with poor prognosis and chemosensitivity in gastric cancer patients. Gastric Cancer 19: 789-797, 2016.

50. An W, Kim J and Roeder RG: Ordered cooperative functions of PRMT1, p300, and CARM1 in transcriptional activation by $\mathrm{p} 53$. Cell 117: 735-748, 2004.

51. Reintjes A, Fuchs JE, Kremser L, Lindner HH, Liedl KR, Huber LA and Valovka T: Asymmetric arginine dimethylation of RelA provides a repressive mark to modulate $\mathrm{TNF} \alpha / \mathrm{NF}-\kappa \mathrm{B}$ response. Proc Natl Acad Sci USA 113: 4326-4331, 2016. 